\title{
DIRETRIZES CURRICULARES NACIONAIS E A EDUCAÇÃO FÍSICA: LEVANTAMENTO DAS PRODUÇÕES ACADÊMICAS E ÇIENTÍFICAS DOS ÚLTIMOS 10 ANOS $^{1}$
}

\author{
Andreia Cristina Metzner \\ Centro Universitário UNIFAFIBE, Bebedouro, São Paulo, Brasil \\ Juliana Cesana \\ Centro Universitário da Fundação Educacional de Barretos, Barretos, São Paulo, Brasil \\ Alexandre Janotta Drigo \\ Universidade Estadual Paulista Júlio de Mesquita Filho, Rio Claro, São Paulo, Brasil
}

\begin{abstract}
Resumo
O objetivo desse estudo foi identificar e analisar as produções científicas e acadêmicas produzidas nos últimos dez anos sobre as Diretrizes Curriculares Nacionais na área de Educação Física. As bases de dados consultadas foram: P@rthenon e Biblioteca Digital Brasileira de Teses e Dissertações. Utilizamos dois termos de busca "Diretrizes Curriculares Nacionais" e "Educação Física". Foram localizados 06 artigos, 18 dissertações e 5 teses, no período de 2006 a 2015. Notamos uma grande concentração de trabalhos nos últimos cinco anos. Os assuntos relacionados as Diretrizes Curriculares Nacionais e a Educação Física envolveram prioritariamente as questões de saúde e currículo. Os resultados apontam a necessidade de revigoramento da produção acadêmica e científica sobre o tema.
\end{abstract}

Palavras-chave: Educação Física. Diretrizes Curriculares Nacionais. Legislação.

\section{Introdução}

O presente estudo teve como objetivo identificar e analisar as produções científicas e acadêmicas realizadas nos últimos dez anos sobre as Diretrizes Curriculares Nacionais de formação na área de Educação Física.

Esta discussão se faz importante diante das recentes mudanças ocorridas nos âmbitos da formação e do campo de atuação da Educação Física, uma vez que esta é uma profissão regulamentada há apenas 18 anos, e os embates pela consolidação de sua área de intervenção ainda se fazem presentes.

No sentido de compreensão da dinâmica pela consolidação profissional da Educação Física, cabe lembrar que a sua profissionalização vem sendo debatida de forma pontual desde os anos 1980 com o advento da Resolução 03/87 quando se criou o curso de Bacharelado na área, estabelecendo pela primeira vez a possibilidade de haverem duas formações distintas ${ }^{2}$,

\footnotetext{
${ }^{1} \mathrm{O}$ presente trabalho não contou com apoio financeiro de nenhuma natureza para sua realização.

${ }^{2}$ As diretrizes de formação anteriores (Decreto-Lei n ${ }^{\circ} 1.212$ de 1939; Decreto-Lei no 8.270 de 1945; e Resolução nº69 de 1969) contemplavam apenas a possibilidade de cursos de Licenciatura em Educação Física, sendo que a formação para atuação extraescolar se tornou preocupação apenas na década de 1980.
} 
como aponta em seu Art. 1: "a formação dos profissionais de Educação Física será feita em curso de graduação que conferirá o título de Bacharel e/ou Licenciado em Educação Física" (BRASIL, 1987, p.01).

Podemos dizer que esse documento alterou a trajetória da Educação Física ao apontar, com maior clareza, que ela é uma área de conhecimento e também um campo profissional que tem atuação nos âmbitos escolar e extraescolar. Sendo assim, nota-se que as condições possibilitadas por essa Resolução provocaram mudanças na formação do profissional de Educação Física, dentre elas a elaboração de uma estrutura curricular que atendesse às duas grandes áreas - Licenciatura e Bacharelado (OLIVEIRA, 2006), e para as quais havia pouca distinção, entendendo-se a licenciatura como uma área de ampla atuação e o bacharelado como uma formação restrita à atuação extraescolar. ${ }^{3}$

Entretanto, no início dos anos 2000 houve uma grande reformulação no entendimento da formação para professores da Educação Básica no Brasil, que culminou no ano de 2002 com as Resoluções CNE/CP 01 e 02 (BRASIL, 2002), com o intuito de fortalecer o professor que atua neste nível de ensino. Esta nova diretriz das licenciaturas trouxe muitas alterações sem precedentes para todas as áreas das disciplinas escolares, inclusive a Educação Física, sendo que no entendimento da nova Lei a formação de professores da Educação Básica deve obedecer à base comum, sendo sua atuação restritiva ao âmbito escolar, como podemos evidenciar no seu Artigo $1^{\circ}$ :

As Diretrizes Curriculares Nacionais para a Formação de Professores da Educação Básica, em nível superior, em curso de licenciatura, de graduação plena, constituem-se de um conjunto de princípios, fundamentos e procedimentos a serem observados na organização institucional e curricular de cada estabelecimento de ensino e aplicam-se a todas as etapas e modalidades da educação básica (BRASIL, 2002).

E é neste momento histórico, em função das Diretrizes Curriculares Nacionais específicas para a formação em cursos de Licenciatura, que se estabelece uma cisão pontual em relação às formações de licenciados e bacharéis em Educação Física e seus respectivos campos de atuação, escolar e extraescolar. Tal divisão fica posteriormente reafirmada na Resolução $\mathrm{n}^{\circ} 07$ de 2004, que foi promulgada com o intuito de instituir as Diretrizes Curriculares Nacionais (DCNs) para o curso de graduação em Educação Física, entendendo-se o termo graduação como homologo ao bacharelado, já que esta resolução define a formação em Educação Física para o âmbito extraescolar, onde estabelece que as orientações específicas para a licenciatura plena em Educação Física, encontram-se definidos nos termos das Diretrizes Curriculares Nacionais para a Formação de Professores da Educação Básica, ou seja, a Resolução no 02/2002 (BRASIL, 2004a, Art. $1^{\circ}$ ).

A Resolução supramencionada aponta que "o Professor da Educação Básica, licenciatura plena em Educação Física, deverá estar qualificado para a docência deste componente curricular na educação básica, tendo como referência a legislação própria do Conselho Nacional de Educação" (BRASIL, 2004a, p.01-02), entendendo-se, portanto, que a formação do professor de Educação Física deve ser concebida, planejada e organizada a partir das Resoluções propostas pelo Conselho Nacional de Educação.

Ainda em relação à construção legal da formação de professores no Brasil, temos atualmente mais uma mudança curricular, sendo que no ano de 2015 foi promulgada a Resolução $n^{\circ} 2$ do Conselho Nacional de Educação (BRASIL, 2015). A presente Resolução define

\footnotetext{
${ }^{3}$ Cabe lembrar que à época a Educação Física ainda não era uma profissão regulamentada, sendo que a instrução de atividades físicas e esportivas fora do ambiente escolar podia ser realizada por qualquer pessoa, sem necessidade de formação ou preparação específicas.
} 
as Diretrizes Curriculares Nacionais para a formação em nível superior de Profissionais da Educação Básica (cursos de licenciatura, cursos de formação pedagógica para graduados e cursos de segunda licenciatura) e para a formação continuada, porém mantendo boa parte do perfil curricular da resolução anterior (Res. 02/2002). Para atender as especificações deste documento, as Instituições de Ensino Superior necessitam adequar a estrutura e o currículo dos cursos atualmente oferecidos até 2017.

Desta forma podemos evidenciar que tanto as formações, como as definições das respectivas áreas de atuação do profissional de Educação Física tiveram mudanças legislativas cruciais nas últimas duas décadas, sendo que as diversas alterações do currículo de formação dos seus profissionais certamente acarretaram impactos tanto nos seus perfis, quanto em suas competências profissionais ao longo do tempo. Faz-se necessário, portanto, analisar o comportamento da Academia em relação a tais alterações, no sentido de compreender o impacto gerado por tais mudanças na reorganização da área e na formação de seus profissionais.

\section{Método}

O presente estudo trata-se de uma Pesquisa Bibliográfica de caráter exploratório com uso exclusivo de bases de dados eletrônicas. Os estudos exploratórios têm como finalidade familiarizar-se com o objeto de estudo para obter uma nova percepção a seu respeito e descobrir novas ideias (MATTOS; ROSSETO JÚNIOR; BLECHER, 2004).

Para a realização do levantamento das produções científicas sobre as Diretrizes Curriculares Nacionais e Educação Física utilizamos as seguintes bases de dados:

- P@rthenon que realiza busca no conteúdo acessado via portal da CAPES e no conjunto de fontes assinadas pela UNESP, e;

- Biblioteca Digital Brasileira de Teses e Dissertações (BDTD) que é uma interface que reúne teses e dissertações defendidas no Brasil em um único portal de busca.

O P@rthenon foi utilizado para mapear os artigos publicados e a BDTD para buscar as publicações no formato de teses e dissertações. Utilizamos na busca dos trabalhos os seguintes descritores: "Diretrizes Curriculares Nacionais" e "Educação Física", bem como, a combinação desses termos. Restringimos nossa busca a estes descritores, pois o intuito do estudo foi de investigar especificamente a produção acadêmico científica ligada à legislação de formação superior em Educação Física e seus possíveis desdobramentos. Não foi feita busca de artigos utilizando os descritores correspondentes em outro idioma. Foram selecionados os trabalhos publicados entre 2006 e 2015, ou seja, os últimos 10 anos.

A análise inicial foi feita com base nos títulos e nos resumos com intuito de verificar se os artigos/dissertações/teses se enquadravam nos seguintes critérios de inclusão: estudos publicados no período estabelecido para busca (últimos dez anos), com texto completo disponível, com a temática central envolvendo simultaneamente as Diretrizes Curriculares Nacionais e a Educação Física. Os estudos que não se enquadravam nesses critérios e os textos em duplicata foram excluídos. Em seguida, os estudos foram examinados na íntegra buscando coletar as informações referentes ao ano de publicação, revista/instituição, e temática central.

\section{Resultados}

A primeira etapa do estudo envolveu a busca de artigos científicos com a utilização do termo "Diretrizes Curriculares Nacionais". Essa etapa resultou em 125 artigos. Em um segundo levantamento, ao utilizarmos os descritores "Diretrizes Curriculares Nacionais" AND "Educação Física" foram identificados apenas 6 estudos. A tabela 1 apresenta os artigos distribuídos por ano e revista. 
Tabela 1 - Artigos distribuídos por ano e por revista

\begin{tabular}{lccccccccccc}
\hline REVISTAS & 06 & 07 & 08 & 09 & 10 & 11 & 12 & 13 & 14 & 15 & Total \\
\hline Interface & - & - & - & - & - & - & - & 1 & - & 1 & 2 \\
Movimento & - & - & - & - & - & - & 1 & - & - & - & 1 \\
Ensaio & - & - & 1 & - & - & - & - & - & - & - & 1 \\
$\begin{array}{l}\text { Pensar a Prática } \\
\text { Revista Brasileira em }\end{array}$ & 1 & - & - & - & - & - & - & - & - & - & 1 \\
Promoção da Saúde & - & - & - & - & - & - & 1 & - & - & - & 1 \\
TOTAL GERAL & 1 & --- & 1 & --- & --- & --- & 2 & 1 & --- & 1 & 6 \\
\hline
\end{tabular}

Fonte: Elaboração Própria

Conforme a Tabela 1, podemos notar que os artigos estão concentrados nos últimos 4 anos (2012, 2013, 2014 e 2015). A Revista Interface possui o maior número de artigos (n=2), seguida das demais revistas que possuem 1 publicação cada.

No que diz respeito aos temas abordados, os dados apresentados na tabela 2 apontam três tópicos, são eles: Saúde, Currículo e Avaliação.

Tabela 2 - Temas abordados nos artigos

\begin{tabular}{ll}
\hline TEMAS & TOTAL \\
\hline Saúde & 03 \\
Currículo & 02 \\
Avaliação & 01 \\
TOTAL GERAL & $\mathbf{0 6}$ \\
\hline
\end{tabular}

Fonte: Elaboração Própria

O tema saúde foi o que teve maior quantidade de artigos publicados ( $\mathrm{n}=03$ ). A Revista Brasileira em Promoção da Saúde publicou um estudo sobre as mudanças ocorridas a partir da instituição das Diretrizes Curriculares Nacionais para os cursos de graduação no campo da saúde (HOLANDA, ALMEIDA e HERMETO, 2012)

Os dois artigos publicados na Revista Interface também estão relacionados a área da saúde, visando identificar as contribuições do PET-Saúde da Família à formação dos profissionais de saúde (SANTANA MADRUGA et. al, 2015) e discutir a inserção do profissional de Educação Física na atenção primária à saúde (FALCI e BELISÁRIO, 2013).

A temática sobre Currículo aparece em segundo lugar $(n=02)$. A pesquisa publicada na Revista Movimento analisa o processo de reestruturação curricular dos cursos de graduação a partir de meados da década de 1990 e, em etapa subsequente, como este ocorre na Educação Física, considerando o processo de elaboração das Diretrizes Curriculares Nacionais (LEMOS et. al, 2012). Na Revista Pensar a Prática, o artigo versa sobre as Diretrizes Curriculares Nacionais de 2004 e a sua relação com o processo de reforma curricular promovido para os cursos de Licenciatura em Educação Física (MUÑOZ et. al, 2006).

Para finalizar, temos um estudo apresentado na Revista Ensaio que discute as políticas de avaliação dos cursos superiores em Educação Física (SANTOS e SIMÕES, 2008).

Após o levantamento dos artigos, realizamos a busca na Biblioteca Digital Brasileira de Teses e Dissertações. O primeiro mapeamento utilizando o descritor "Diretrizes Curriculares Nacionais" trouxe como resultado 439 estudos. Após combinarmos esse descritor com o termo "Educação Física" encontramos um total de 23 pesquisas, sendo 18 dissertações e 5 teses. A tabela 3 apresenta o resultado dessa busca. 
Tabela 3 - Teses e Dissertações distribuídas por instituição

\begin{tabular}{llll}
\hline INSTITUIÇÃO & TESE & DISSERTAÇÃO & TOTAL \\
\hline UFSC & & & \\
UNICAMP & --- & 03 & 03 \\
UFBA & 01 & 02 & 03 \\
UFPEL & 01 & 01 & 02 \\
UFRGS & 01 & 01 & 02 \\
UFSCAR & --- & 02 & 02 \\
UNB & 01 & 01 & 02 \\
UNESP & --- & 02 & 02 \\
UFG & --- & 02 & 02 \\
UERJ & --- & 01 & 01 \\
UFES & 01 & --- & 01 \\
UFRN & --- & 01 & 01 \\
UNOESTE & --- & 01 & 01 \\
TOTAL GERAL & --- & 01 & 01 \\
\hline
\end{tabular}

Fonte: Elaboração Própria

Sobre as publicações de teses e dissertações, verificamos que o número de dissertações $(\mathrm{n}=18)$ sobrepõe- se ao de tese $(\mathrm{n}=05)$. $\mathrm{E}$ as instituições que tiveram um maior número de trabalhos foram a UFSC e UNICAMP, contemplando 3 estudos cada uma.

Em relação a distribuição das pesquisas durante os últimos dez anos, notamos que o período de 2011 à 2015 houve um maior número de publicações. Nesse período foi desenvolvido quase o dobro de trabalhos $(\mathrm{n}=15)$ comparado ao período anterior de 2006 a 2010 $(\mathrm{n}=08)$, conforme pode ser visualizado na tabela 4 .

Tabela 4 - Teses e Dissertações distribuídas por ano e por instituição

\begin{tabular}{lccccccccccc}
\hline INSTITUIÇÃO & $\mathbf{0 6}$ & $\mathbf{0 7}$ & $\mathbf{0 8}$ & $\mathbf{0 9}$ & $\mathbf{1 0}$ & $\mathbf{1 1}$ & $\mathbf{1 2}$ & $\mathbf{1 3}$ & $\mathbf{1 4}$ & $\mathbf{1 5}$ & $\begin{array}{c}\text { TO- } \\
\text { TAL }\end{array}$ \\
\hline UFSC & & & & & & & & & & & \\
UNICAMP & 1 & - & - & - & - & 1 & 1 & - & - & - & 03 \\
UFBA & - & 1 & - & 1 & - & - & - & - & - & - & 02 \\
UFPEL & - & - & - & - & - & - & 2 & - & - & - & 02 \\
UFRGS & - & - & - & - & 1 & - & - & 1 & - & - & 02 \\
UFSCAR & - & - & 1 & - & - & - & - & 1 & - & - & 02 \\
UNB & - & - & 1 & - & 1 & - & - & - & - & - & 02 \\
UNESP & - & 1 & - & - & - & - & - & - & - & 1 & 02 \\
UFG & - & - & - & - & - & 1 & - & - & - & - & 01 \\
UERJ & - & - & - & - & - & - & - & - & 1 & - & 01 \\
UFES & - & - & - & - & - & - & 1 & - & - & - & 01 \\
UFRN & - & - & - & - & - & 1 & - & - & - & - & 01 \\
UNOESTE & - & - & - & - & - & - & - & - & 1 & - & 01 \\
TOTAL GERAL & $\mathbf{1}$ & $\mathbf{2}$ & $\mathbf{2}$ & $\mathbf{1}$ & $\mathbf{2}$ & $\mathbf{4}$ & $\mathbf{4}$ & $\mathbf{2}$ & $\mathbf{2}$ & $\mathbf{3}$ & $\mathbf{2 3}$ \\
\hline
\end{tabular}

Fonte: Elaboração Própria

As pesquisas desenvolvidas nas teses e dissertações versam sobre as Diretrizes Curriculares Nacionais e a sua relação com cinco principais temas: Currículo, Formação Profissional, Educação Infantil, Saúde e Formação Continuada. 
O tema que abarca o maior número de estudos é o Currículo, sendo identificado em 03 teses e 10 dissertações conforme mostra a tabela 5.

Tabela 5 - Temas abordados nas Teses e Dissertações

\begin{tabular}{lccc} 
TEMAS & TESES & DISSERTAÇÕES & TOTAL \\
\hline Currículo & 03 & 10 & 13 \\
Formação Profissional & 02 & 03 & 05 \\
Educação Infantil & & 02 & 02 \\
Saúde & & 02 & 02 \\
Formação Continuada & $\mathbf{0 5}$ & 01 & 01 \\
TOTAL GERAL & & $\mathbf{1 8}$ & $\mathbf{2 3}$ \\
\hline
\end{tabular}

Fonte: Elaboração Própria

O Currículo é abordado nas teses e dissertações sob diferentes vertentes: O currículo do Ensino Médio, a prática como componente curricular, as reformas curriculares nos cursos de Licenciatura e Bacharelado, a reorganização do estágio supervisionado no currículo e o lugar da pesquisa no currículo dos cursos de formação inicial.

O tema da Formação Profissional totalizou cinco estudos, sendo duas teses e três dissertações. Essas pesquisas tiveram como foco o estudo da crise epistemológica da Educação Física, as consequências das Diretrizes Curriculares Nacionais na formação do profissional de Educação Física, a conexão entre o perfil e concepção dos professores em relação às DCNs e a formação profissional sob a perspectiva da saúde pública a partir das Diretrizes para a graduação em Educação Física.

A Educação Infantil foi destaque em duas dissertações. Um dos estudos teve como foco o histórico das propostas educacionais voltadas à Educação Física na Educação Infantil e o outro analisou os documentos oficiais da Educação Infantil prescritos pelos órgãos centrais dos sistemas de ensino.

$\mathrm{Na}$ área da saúde foram encontradas duas dissertações que buscaram gerar informações sobre o reconhecimento dos profissionais de Educação Física como membros da equipe de saúde por meio das Diretrizes Curriculares Nacionais e o trato do conhecimento em relação à Educação Física e saúde nos currículos de formação inicial.

Para finalizar, encontramos uma dissertação que envolveu o tema Formação Continuada em serviço com o intuito de analisar a atuação dos professores de Educação Física, sua participação, reações e aproveitamento das atividades desenvolvidas durante essa formação.

\section{Discussão}

Os resultados do presente estudo dão ênfase a pontos importantes sobre as questões que envolvem as Diretrizes Curriculares Nacionais na área de Educação Física que merecem destaques, tais como: as datas de publicações, principais periódicos, instituições de ensino superior que pesquisaram sobre esse tema e os assuntos abordados nos estudos.

No que concerne a data de publicação das pesquisas, observamos que o número de produções a respeito das Diretrizes Curriculares Nacionais ao longo dos últimos dez anos aumentou na segunda metade do período pesquisado (2011 a 2015) tanto em relação aos artigos publicados como em relação as teses e dissertações.

Os estudos e produções acadêmicas na área de Educação Física vem crescendo nos últimos anos. Essa expansão é decorrente do aumento do número de programas de pósgraduação, de grupos de pesquisa, da produção intelectual, de congressos e eventos na área. (KOKUBUN, 2003). Atualmente, podemos dizer que "área de educação física deixou de ser 
apenas uma área de prática pedagógica ou de aplicação de conceitos provindos de outras áreas acadêmicas para tornar-se área de produção de conhecimento científico" (DAÓLIO, 2007, p.50).

No caso das pesquisas relacionadas as Diretrizes Curriculares Nacionais, acreditamos que o crescimento no número de publicações nos últimos 5 anos aconteceu, principalmente, porque após a promulgação das Resoluções 01 e 02/CNE/2002 os cursos tiveram um período de adequação de 2 anos, e depois foi prorrogado por mais um tempo até 15 de outubro de 2005 (BRASIL, 2004b). Essas Resoluções promoveram a primeira mudança curricular na área de Educação Física com grandes proporções. Dentre as principais alterações podemos citar que os cursos de Licenciatura passaram a formar professores que atuariam exclusivamente na Educação Básica (Educação Infantil, Ensino Fundamental e Ensino Médio), restringindo o campo de atuação do licenciado em Educação Física para o ambiente escolar (BRASIL, 2002a), e a definição da carga horária dos cursos de licenciatura que passou a ter no mínimo 2800 (duas mil e oitocentas) horas, distribuídos em 200 dias letivos/ano, integralizada em no mínimo 3 anos letivos (BRASIL, 2002b). Desta forma, a expansão das produções científicas envolvendo essa temática culminou com as primeiras turmas formadas nesse novo currículo.

Em relação a distribuição dos artigos por revistas encontramos resultados bem similares. Das cinco revistas mapeadas, quatro publicaram um artigo cada e apenas a Revista Interface teve duas publicações.

$\mathrm{Na}$ busca realizada nas teses e dissertações também não houve nenhum grande destaque. Nas treze instituições de ensino expostas, o número de pesquisas foi equilibrado, girando em torno de duas ou uma defesa. Apenas a UFSC e UNICAMP apresentou três estudos cada uma.

Outro resultado que merece evidência é o tema abordado em cada um desses estudos. Dos 06 artigos científicos, 03 pesquisaram sobre a questão da Saúde. Já nas teses e dissertações, a temática "Currículo" se sobressaiu sobre os demais assuntos pesquisados, totalizando 13 estudos.

Na última década, "políticas públicas de educação e saúde promovidas em parceria com o Ministério da Educação (MEC) e o Ministério da Saúde (MS) sinalizaram uma reforma curricular imprescindível nos cursos de graduação da área de saúde”. (HOLANDA, ALMEIDA e HERMETO, 2012, p.389).

Na área de Educação Física, a questão da saúde ganhou força a partir do momento que o Conselho Nacional de Saúde, em 1997, por meio da Resolução $n^{\circ} 218$, afirma a importância da ação interdisciplinar realizadas por diferentes profissionais no campo da saúde e reconhece os profissionais de Educação Física como membros dessa equipe interdisciplinar juntamente com mais doze categorias: Assistentes Sociais, Biólogos, Enfermeiros, Farmacêuticos, Fisioterapeutas, Fonoaudiólogos, Médicos, Médicos Veterinários, Nutricionistas, Odontólogos, Psicólogos e Terapeutas Ocupacionais (BRASIL, 1997).

As Diretrizes Curriculares Nacionais para a graduação em Educação Física instituídas pela Resolução $\mathrm{n}^{\circ} 7$, promulgada em março de 2004 , vem de encontro aos dizeres da Resolução $\mathrm{n}^{\circ} 218$ supramencionada, ao reforçar a necessidade de formar profissionais de Educação Física capazes de intervir "nos campos da prevenção, promoção, proteção e reabilitação da saúde", bem como, "participar, assessorar, coordenar, liderar e gerenciar equipes multiprofissionais de discussão, de definição e de operacionalização de políticas públicas e institucionais nos campos da saúde" (BRASIL, 2004a, Art. 06, $\S 1^{\circ}$ ). Dessa forma, os cursos de formação de Educação Física tiveram que se adequar a essas normativas incorporando em sua grade 
curricular disciplinas, projetos, intervenções, discussões, ações, etc. direcionadas a questão da saúde.

Essas mudanças refletiram nas produções acadêmicas e científicas na busca de legitimar o papel do profissional de Educação Física nesse novo campo de atuação. Por isso, nos últimos anos os artigos publicados tiveram como temática central a saúde.

Em relação ao Currículo, que foi o tema principal que sustentou a maioria das teses e dissertações, acreditamos que esta é uma problemática que se modifica ao longo dos tempos e que as reformulações legislativas são as principais fontes que impulsionam essas modificações.

Sacristán (2000) aponta que o início da construção das matrizes curriculares acontece por meio das políticas públicas. São as políticas educativas que prescrevem critérios mínimos e orientações curriculares para serem seguidos dentro do sistema educativo. O autor denomina este nível curricular como "currículo prescrito ou oficial". O currículo prescrito é apresentado no âmbito escolar por meio dos documentos oficiais, como Decretos, Resoluções, Diretrizes, Leis, etc.

As Diretrizes Curriculares Nacionais são normas obrigatórias que orientam o planejamento curricular das instituições de ensino, estabelecidas pelo Conselho Nacional de Educação. E "definir as competências necessárias para o desenvolvimento da atuação profissional e tê-las como "norte" na organização das propostas pedagógicas, institucionais e gestoras, são as bases para a concepção das diretrizes" (QUARANTA, 2011, p.69).

Dessa forma, a partir das considerações de Sacristán (2000), podemos dizer que as Diretrizes Curriculares Nacionais fazem parte do currículo prescrito.

Em termos gerais, currículo prescrito é:

Um aspecto específico da política educativa, que estabelece a forma de selecionar, ordenar e mudar o currículo dentro do sistema educativo, tornando claro o poder e a autonomia que diferentes agentes têm sobre ele, intervindo, dessa forma, na distribuição do conhecimento dentro do sistema escolar e incidindo na prática educativa, enquanto apresenta o currículo a seus consumidores, ordena seus conteúdos e códigos de diferente tipo (SACRISTÁN, 2000, p.109).

A intervenção política que as Diretrizes Curriculares Nacionais desempenham sobre o currículo nas diversas áreas do conhecimento é decisiva para o delineamento dos cursos e, consequentemente, reflete na qualidade do ensino. Por isso, acreditamos que a maioria das teses e dissertações analisadas discutiu aspectos específicos presentes no currículo dos cursos de Educação Física, como por exemplo, o estágio supervisionado, práticas pedagógicas, pesquisa científica, reorganização curricular, etc., com o intuito de elucidar o lugar ocupado por cada um desses componentes.

As outras temáticas encontradas nos artigos e nas teses/dissertações, apesar de pouca magnitude comparada as publicações realizadas sobre esses dois assuntos supramencionados (Saúde e Currículo), também merecem destaques em nossa discussão, pois sugerem que as questões que envolvem a formação profissional, Educação Infantil, formação continuada e avaliação sejam melhor investigadas.

As possibilidades de pesquisas que vertem sobre as Diretrizes Curriculares Nacionais são muitas. Por isso, acreditamos que nos próximos anos mais estudos e investigações pertinentes serão efetuados e, por consequência, o número de publicações aumentará.

\section{Conclusão}

Apesar de as Diretrizes Curriculares Nacionais se configurarem como um dos temas de estudo que esteve em grande evidência na última década, tendo 125 artigos e 439 te- 
ses/dissertações publicadas sobre esse assunto, esta revisão bibliográfica mostrou um número limitado de pesquisas sobre o assunto na área de Educação Física.

As tendências contemporâneas e as constantes discussões no campo acadêmico e profissional, modificam de tempos em tempos os dizeres presentes nas Diretrizes Curriculares Nacionais de formação, e as orientações propostas nessas Leis são referências para a elaboração dos currículos e devem ser respeitadas por todas as instituições de ensino. Em razão disto, o esperado era que esse assunto fosse intensificado no meio acadêmico.

É importante ressaltar que os artigos, teses e dissertações apresentados nessa pesquisa foram extraídos a partir de descritores específicos, referentes a período de tempo prédeterminado e duas bases de dados (P@arthenon e BDTD), que apesar de abrangerem um grande número de pesquisas, não alcançam a totalidade de trabalhos publicados sobre a temática abordada. Portanto, apontamos estes fatores como limitação do estudo.

Mas, apesar dessa limitação, por meio desta investigação foi possível traçar um panorama dos últimos dez anos acerca das pesquisas sobre as Diretrizes Curriculares Nacionais para a Educação Física. Sugerimos estudos mais aprofundados a respeito dessa temática para validar os dados coletados, bem como, conduzir novas descobertas, uma vez que a partir do levantamento realizado, notamos a carência de produções acadêmicas e científicas nessa área.

\title{
NATIONAL CURRICULAR DIRECTIVES AND PHYSICAL EDUCATION: A RE- VIEW OF ACADEMIC AND SCIENTIFIC PRODUCTION OF THE LAST 10 YEARS
}

\begin{abstract}
The aim of this study was to identify and analyze the scientific and academic productions produced in the last ten years on the National Curricular Directives in the area of Physical Education. The databases consulted were: P@rthenon and Biblioteca Digital Brasileira de Teses e Dissertações. We used two search terms "National Guidelines" and "Physical Education". Were located 06 articles, 18 dissertations and 5 theses, in the period from 2006 to 2015. We noted a large concentration of articles in the last five years. The issues the National Curriculum Guidelines and Physical Education primarily involved questions about health and curriculum. The results show the need for strengthening of academic and scientific literature on this topic.
\end{abstract}

Keywords: Physical Education. National Curricular Directives. Legislation.

\section{DIRECTIVAS CURRICULARES NACIONALES Y LA EDUCACIÓN FÍSICA: LA ELIMINACIÓN DE ACADÉMICA Y CIENTÍFICA DE PRODUCCIÓN LOS ÚLTI- MOS 10 AÑOS}

\begin{abstract}
Resumen
El objetivo de este estudio fue identificar y analizar las producciones científicas y académicas producidas en los últimos diez años en las Directivas Curriculares Nacionales en el área de Educación Física. Las bases de datos consultadas fueron: P @ rthenon y la Biblioteca Digital Brasileña de Tesis y Disertaciones. Se han utilizado dos términos de búsqueda "Directrices Nacionales" y "Educación Física". 06 artículos, 18 disertaciones y tesis 5 se encuentran en el período de 2006 a 2015. Se observó una gran concentración de puestos de trabajo en los últimos cinco años. Los problemas de las Directrices Curriculares Nacionales de Educación Física y problemas de salud principalmente involucrado y del plan de estudios. Los resultados muestran la necesidad de fortalecer la literatura académica y científica sobre el tema.
\end{abstract}


Palabras clave: Educación Física. Directivas Curriculares Nacionales. La legislación.

\section{Referências}

BRASIL. Conselho Nacional de Educação. Resolução no 2, de $1^{\circ}$ de julho de 2015. Define as Diretrizes Curriculares Nacionais para a formação inicial em nível superior (cursos de licenciatura, cursos de formação pedagógica para graduados e cursos de segunda licenciatura) e para a formação continuada. Brasília, DF, 2015.

BRASIL. Conselho Nacional de Educação. Resolução no 7, de 31 de março de 2004. Institui as Diretrizes Curriculares Nacionais para os cursos de graduação em Educação Física, em nível superior de graduação plena. Brasília, DF, 2004a.

BRASIL. Conselho Nacional de Educação. Resolução no 2, de 27 de agosto de 2004. Adia o prazo previsto no art. 15 da Resolução CNE/CP 1/2002, que institui Diretrizes Curriculares Nacionais para a Formação de Professores da Educação Básica, em nível superior, curso de licenciatura, de graduação plena. Brasília, DF, 2004b.

BRASIL. Conselho Nacional de Educação. Resolução CNE/CP nº1, de 18 de fevereiro de 2002. Institui Diretrizes Curriculares Nacionais para a Formação de Professores da Educação Básica, em nível superior, curso de licenciatura, de graduação plena. Brasília, DF, 2002a.

BRASIL. Conselho Nacional de Educação. Resolução CNE/CP n02, de 19 de fevereiro de 2002. Institui a duração e a carga horária dos cursos de licenciatura, de graduação plena, de formação de professores da Educação Básica em nível superior. Brasília, DF, 2002b.

BRASIL. Conselho Nacional de Saúde. Resolução no 218, de 6 de março de 1997. Reconhece os profissionais de Educação Física como Profissionais da Saúde. Brasília, DF, 1997.

BRASIL. Conselho Nacional de Educação. Resolução no 03, de 16 de junho de 1987. Fixa os mínimos de conteúdo e duração a serem observados nos cursos de graduação em Educação Física (Bacharelado e/ou Licenciatura Plena). Brasília, DF, 1987.

DAÓLIO, J. O ser e o tempo da pesquisa sociocultural em Educação Física. Revista Brasileira de Ciências do Esporte, v. 29, n. 1, 2007.

FALCI, D. M.; BELISÁRIO, S. A. A inserção do profissional de educação física na atenção primária à saúde e os desafios em sua formação. Interface-Comunicação, Saúde, Educação, v. 17, n. 47, p. 885-899, 2013.

HOLANDA, I. C. L. C. de; ALMEIDA, M. M. de; HERMETO, E. M. C. Indutores de mudança na formação dos profissionais de saúde: PRÓ-SAÚDE E PETSAÚDE. Revista Brasileira em Promoção da Saúde, v. 25, n. 4, p.389-392, 2012.

KOKUBUN, E. Pós-graduação em Educação Física no Brasil: indicadores objetivos dos desafios e das perspectivas. Revista Brasileira de Ciências do Esporte, v. 24, n. 2, 2003. 
LEMOS, L. M. et al. As contradições do processo de elaboração das diretrizes curriculares nacionais dos cursos de formação em Educação Física e os movimentos de resistência à submissão ao mercado. Movimento (ESEF/UFRGS), v. 18, n. 3, p. 27-49, 2012.

MATTOS, M. G.; ROSSETO Jr, A. J.; BLECHER, S. Teoria e prática da metodologia da pesquisa em Educação Física: construindo seu trabalho acadêmico - monografia, artigo científico e projeto de ação. São Paulo: Phorte, 2004.

MUÑOZ, G. H. et al. Reforma curricular dos cursos de licenciatura em Educação Física: contribuições dos professores das redes públicas de ensino. Pensar a Prática, v.9, n. 2, p. 231248, 2006.

OLIVEIRA, A. Ap. B. A formação profissional em Educação Física: legislação, limites e possibilidades. In: SOUZA NETO, S.; HUNGER, D. (Org.). Formação profissional em Educação Física: estudos e pesquisas. Rio Claro: Biblioética, 2006. p.17-32.

QUARANTA, A. M. Formação de professores de educação física na modalidade de educação à distância: experiências docentes no estágio supervisionado. 2011. 207f. Dissertação (Mestrado em Educação Física) - Pós-Graduação em Educação Física, Centro de Desportos, Universidade Federal de Santa Catarina, Florianópolis, 2011.

SACRISTÁN, J. G. O currículo: uma reflexão sobre a prática. Porto Alegre: ArtMed, 2000.

SANTANA MADRUGA, L. M. et al. O PET-Saúde da Família e a formação de profissionais da saúde: a percepção de estudantes. Interface-Comunicação, Saúde, Educação, v. 19, n. 1, p. 805-816, 2015.

SANTOS, A. L. P; SIMÕES, A. C. Desafios do ensino superior em educação física: considerações sobre a política de avaliação de cursos. Ensaio: Avaliação e Políticas Públicas em Educação, v. 16, n. 59, p. 259-274, 2008.

Recebido em: 09/07/2016

Revisado em: 10/10/2016

Aprovado em: 10/10/2016

Endereço para correspondência:

acmetzner@hotmail.com

Andreia Cristina Metzner

Centro Universitário UNIFAFIBE

Rua Prof. Orlando França de Carvalho, 325 - Centro

Bebedouro/SP

CEP 14.701-070 